\title{
Temporal sound field fluctuations in the presence of internal solitary waves in shallow water
}

\author{
Boris G. Katsnelson and Valery Grigorev \\ Voronezh University, 1 Universitetskaya Square, Voronezh 394006, Russia \\ katz@phys.vsu.ru,grig@box.vsi.ru \\ Mohsen Badiey \\ College of Marine and Earth Studies, University of Delaware, Newark, Delaware 19716 \\ badiey@udel.edu \\ James F. Lynch \\ Woods Hole Oceanographic Institution, 98 Water Street, MS No. 12, Woods Hole, Massachusetts 02543 \\ jlynch@whoi.edu
}

\begin{abstract}
Temporal variations of intensity fluctuations are presented from the SWARM95 experiment. It is hypothesized that specific features of these fluctuations can be explained by mode coupling due to the presence of an internal soliton moving approximately along the acoustic track. Estimates are presented in conjunction with theoretical consideration of the shallow water waveguide.

(C) 2009 Acoustical Society of America

PACS numbers: 43.30.Bp, 43.30.Dr, 43.30.Es, 43.30.Zk [WS]

Date Received: July 17, 2007 Date Accepted: May 14, 2009
\end{abstract}

\section{Introduction}

Sound intensity fluctuations are observed in the SWARM95 experiment ${ }^{1}$ when an internal soliton (IS), or a train of IS, propagates approximately along an acoustic track with wave crest making an angle of approximately $40^{\circ}$ (Fig. 1). During a part of this experiment, broadband pulses (in the 30-200 Hz band) were radiated every minute from an airgun and received on two vertical line arrays (called NRL-VLA and WHOI-VLA) at a distance of 14-18 km from the sound source. Figure 1 shows a schematic of the source and receiver arrays. The authors reported analysis of the WHOI-VLA results previously ${ }^{2}$ and established that the mechanism dictating the temporal behavior of arriving sound signals is governed by horizontal refraction. Other numerical calculations involved mode coupling. ${ }^{3}$ However, the same mechanism is not adequately depicting the temporal variations observed in the NRL-VLA data, for example, Ref. 3. In this paper, the authors present theoretical estimates, supported by experimental data, stating that specific features of mode coupling are responsible for the intensity fluctuations.

The observed temporal variations during a $1 \mathrm{~h}$ long pulse transmission (1 pulse/min) on the NRL-VLA have the characteristics of (1) predominant frequency in the fluctuations' spectrum below $20 \mathrm{cph}$ for different sound frequencies [shown in Fig. 2(b)], (2) specific arrival time spreading for different modes on the time-frequency (TF) diagram [shown in Fig. 3(b)], and (3) approximately constant correlation time in a wide range of sound frequency [shown in Fig. 4(b)].

To complement our previous results on the WHOI-VLA having distinctly different of acoustic track geometry in relation to the IS direction, ${ }^{1,2}$ and where the temporal variations were due to horizontal refraction, the variations observed on the NRL-VLA can be explained by specific features of the mode coupling. ${ }^{3}$ This mechanism can uniquely describe the behavior of all the pulses that have passed through the IS and were received by the NRL-VLA. A pulse radiated from the airgun is a sum of separate normal modes propagating with separate group velocities. After interaction with the IS located at some distance $R$ from the source, this sum changes and in turn the sound field at the receiver changes (in comparison with unperturbed 


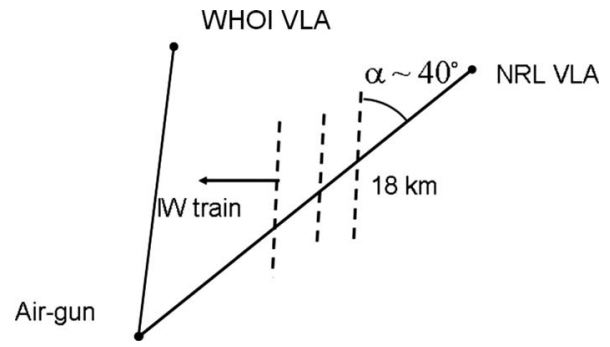

Fig. 1. Experimental schematic of the SWARM95 during Aug. 14, 1995. The directions of acoustic track formed by UD source and NRL and WHOI vertical line arrays are shown with respect to the predominant direction of the internal wave shown by dotted lines; $\alpha$ is the horizontal angle between these directions.

waveguide). For other positions of the moving IS relative to the source/receiver track (or for another geotime), there will be other combinations of modes created, and a different sound field at the receiver is observed. For fixed source-receiver geometry, the authors perceive this variability as temporal fluctuations. Typical frequencies of the fluctuations are about $\sim 1-10 \mathrm{cph}$.
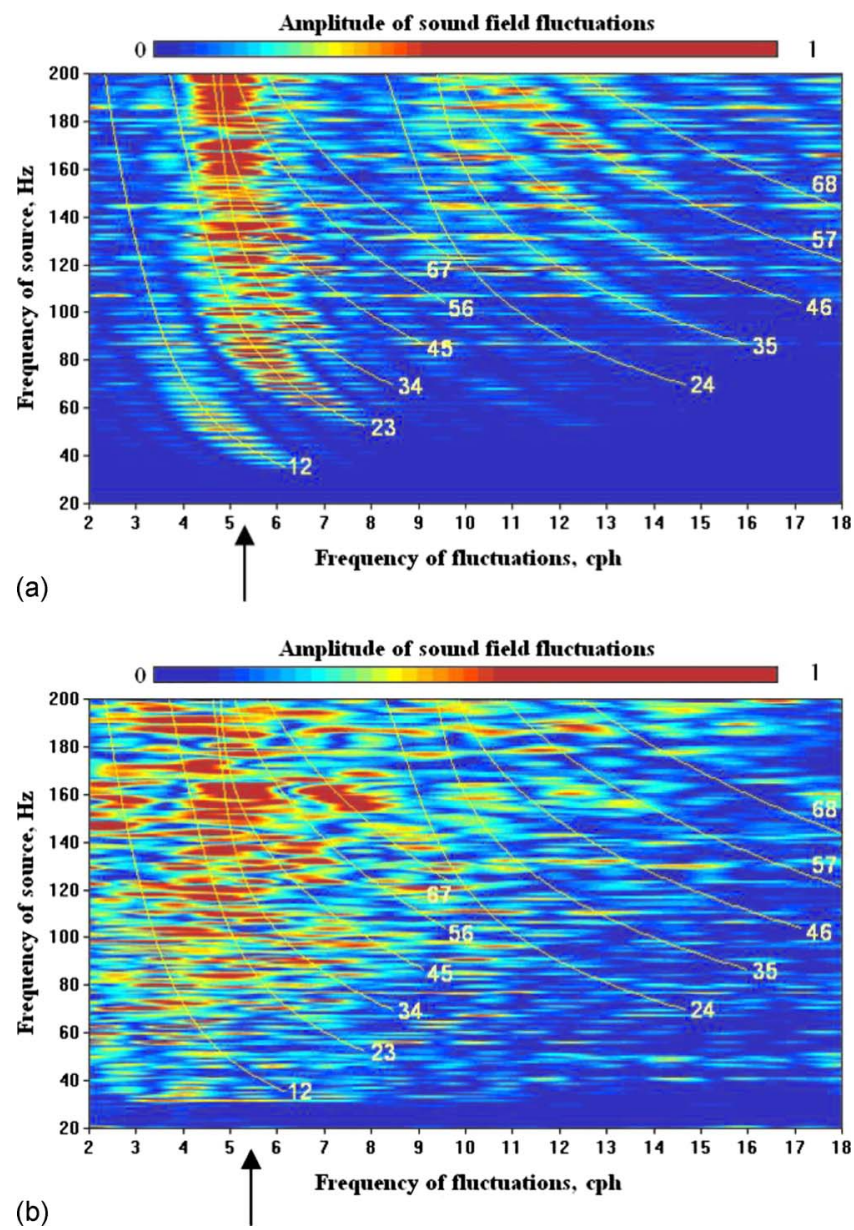

Fig. 2. (Color online) A frequency-frequency $(f F)$ diagram for SWARM95 showing the spectral intensity $G$ of received signal as function of frequency: (a) theory and (b) experiment. 

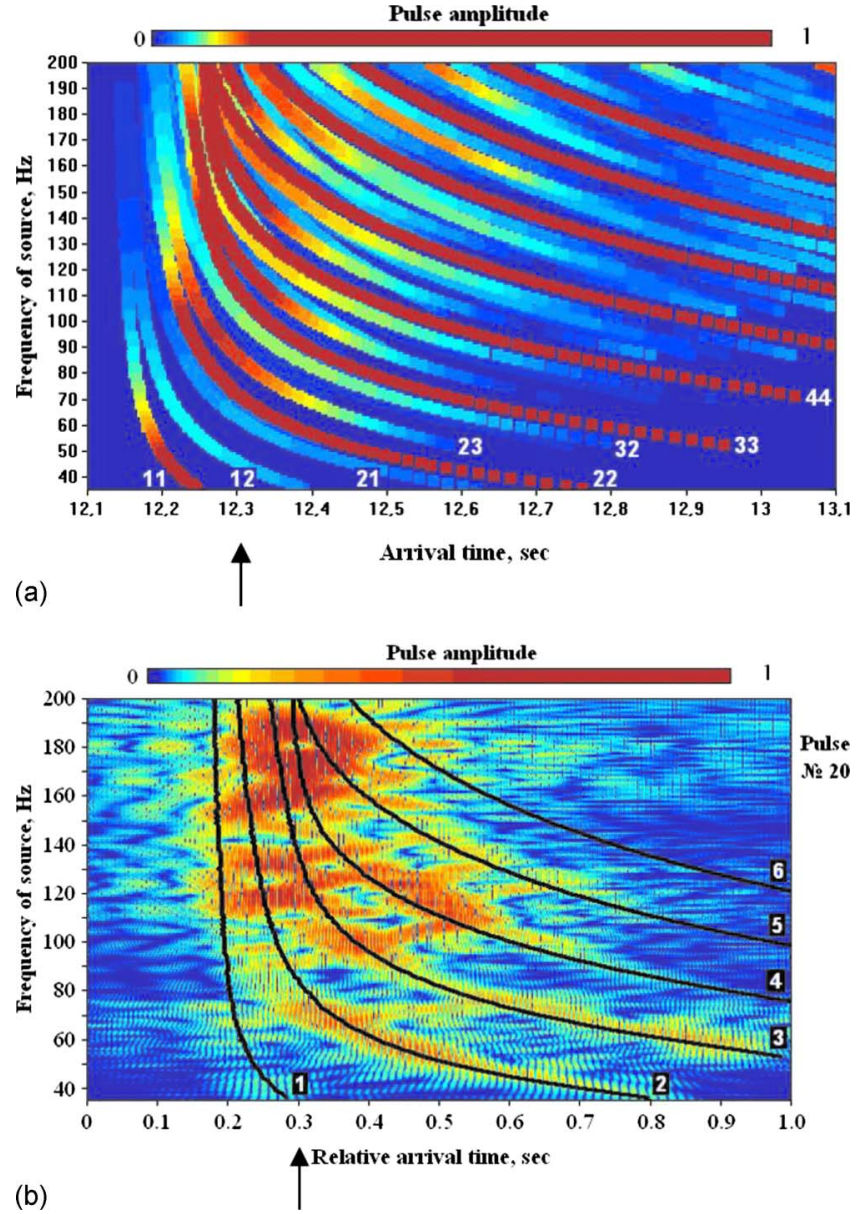

Fig. 3. (Color online) Frequency-time diagram for arrival times. Color scale gives amplitudes of separate modes according to scale above the figures: (a) theory and (b) experiment. Lines 11, 22, etc., in (a) denote positions of modes without coupling (or without a soliton); in (b), these lines are denoted by black curves with numbers 1, 2, 3 , etc.

The specific waveguide features essential for understanding the nature and properties of these temporal fluctuations are (1) a narrow thermocline layer (10-35 m deep, within a water column of $\sim 90 \mathrm{~m}$ total depth), (2) approximately constant soliton shapes and velocities during a few hours $\left(v_{s} \sim 0.5-1 \mathrm{~m} / \mathrm{s}\right)$, and (3) length $\Delta R$ of the IS less than the acoustic track $L(\Delta R$ $\sim 300-1000 \mathrm{~m} \ll L \sim 15-20 \mathrm{~km}$ ). Here, preliminary results in support of the above statement are presented to explain the received temporal variability at the NRL-VLA array.

\section{Waveguide model}

A point source with spectrum $S(\omega)$ was placed at depth $z_{s}$ in a shallow water waveguide. The authors assume the unperturbed waveguide has a constant depth $H$, bottom parameters $c_{1}$ and $\rho_{1}$, and unperturbed sound speed profile $c(z)$. Bottom attenuation is neglected. At the receiver point $(r, z)$ the Fourier decomposition of the sound field pressure is

$$
P(r, z, t)=2 \int_{0}^{\infty} P_{\omega}(r, z) e^{-i \omega t} d \omega .
$$



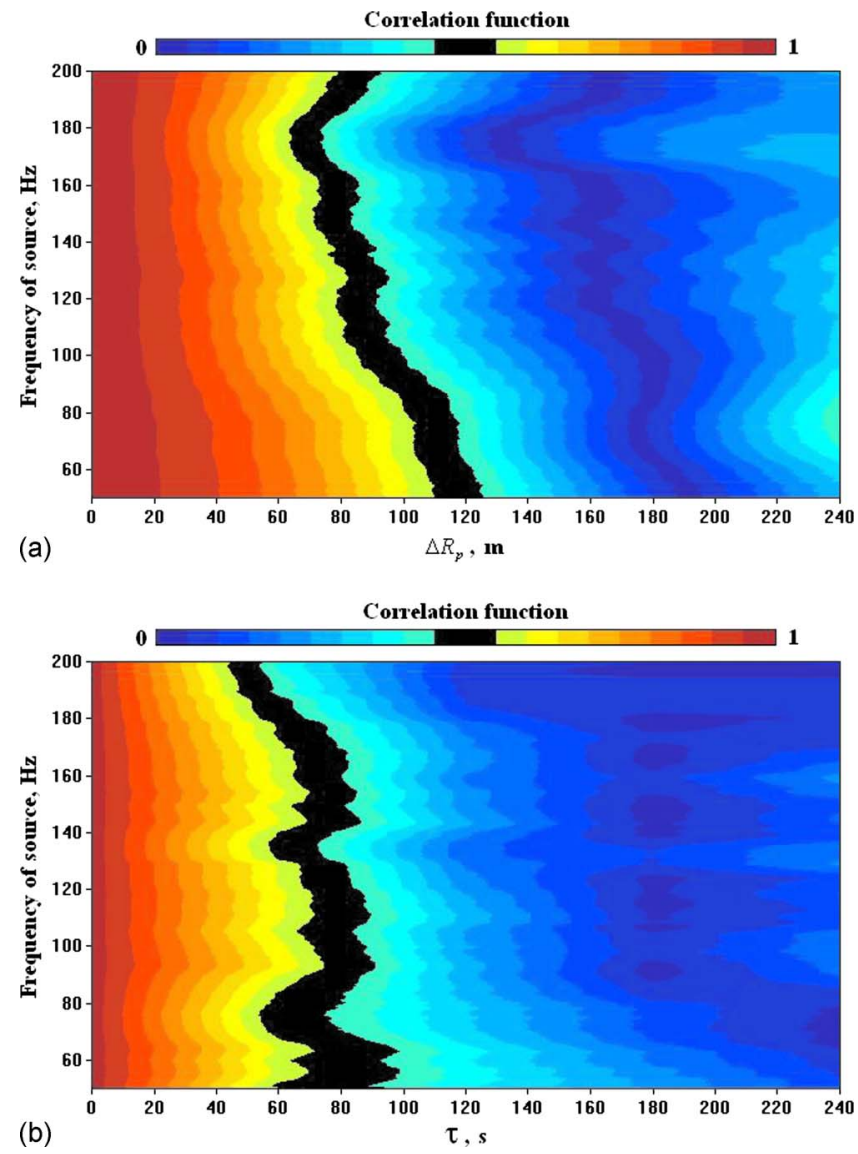

Fig. 4. (Color online) Correlation function for SWARM'95 conditions in color gradation of amplitude (scale is shown); black denotes half level: (a) theory and (b) experiment.

In the absence of perturbations or in the area $r<R$, the amplitude $P_{\omega}(r, z)$ is the following modal decomposition over waveguide modes $\psi_{l}$ (where $q_{l}$ is eigenvalue):

$$
P_{\omega}(r, z)=i S(\omega) \sum_{l} \frac{\psi_{l}\left(z_{s}\right) \psi_{l}(z)}{\sqrt{8 \pi i q_{l} r}} \exp \left(i q_{l} r\right) .
$$

In the presence of the IS moving with constant velocity $v$ (if there is some angle $\alpha$ between the direction of the acoustic track and the wave front of the IS, the actual velocity of the IS is $v_{s}=v \sin \alpha$; see Fig. 1), there is a perturbation of the sound field. At a given moment of time $T$, this perturbation occupies an area at the range $R<r<R+\Delta R$, where $R=v T$, and in this area the authors have a small addition to the sound speed profile, $\delta c(r, z, T)$, which depends on the "slow" time $T$. This moving sound speed perturbation produces fluctuations of the sound field also depending on $T$. After acoustic interaction with the soliton, the authors have another modal decomposition for the sound field for $r>R+\Delta R$. The authors describe this decomposition using $S$-matrix formalism

$$
P_{\omega}(r, z ; R)=\sum_{m}\left[i S(\omega) \sum_{l} \frac{\psi_{l}\left(z_{s}\right) \psi_{m}(z)}{\sqrt{8 \pi i q_{m} r}} S_{m l}(R+\Delta R, \omega) \exp \left(i \Delta q_{m l} R\right)\right] \exp \left(q_{m} r\right),
$$

where $\Delta q_{l m}=q_{l}-q_{m}$, the matrix $\mathbf{S}(r)$ satisfies the equation with "initial" condition 


$$
\frac{d \mathbf{S}}{d r}=\mathbf{W S}, \quad \mathbf{S}(R)=\mathbf{I},
$$

where $\mathbf{I}$ is the unit matrix, and the coupling coefficients in simple perturbation theory are

$$
W_{m l}(r)=i \frac{k^{2} \exp \left[i\left(q_{l}-q_{m}\right) r\right]}{\sqrt{q_{m} q_{l}}} \int_{0}^{H} \frac{\delta c(r, z)}{c} \psi_{m}(z) \psi_{l}(z) d z
$$

Due to $T=R / v$, the authors will simply write $P_{\omega}(T)$ (omitting receiver coordinates). Expression in square brackets in Eq. (3) is effectively the amplitude of the $m$ th mode. The sound field at the receiver equation (3) has several different scales of periodicity as a function of the IS's position $R$, and thus on the time $T$. Since the spatial scales of acoustic variability are determined by the parameter $\Lambda_{l m}=2 \pi /\left|\Delta q_{l m}\right|$, the authors have correspondingly frequencies of temporal variability $\Omega_{l m}=v\left|\Delta q_{l m}\right|$. Typically the value of $\Omega_{l m}$ is $\sim 10^{-4}-2 \times 10^{-3} \mathrm{~Hz}(\sim 1-15 \mathrm{cph})$. Assuming that the strongest coupling interaction is between adjacent modes (close coupling), the authors can write from Eq. (3) a form for the normalized (with respect to the source spectrum) amplitude of mode $m$ created from mode $m+1: A_{m, m+1}=\left|\psi_{m+1}\left(z_{s}\right) \psi_{m}(z) S_{m, m+1} / \sqrt{8 \pi i q_{m}}\right|$. This quantity depends on frequency, and for each pair of coupling modes, the coefficients $A_{m, m+1}$ have their own "optimal" frequency, denoted by $\omega_{\mathrm{opt}}^{m}=\omega_{\mathrm{opt}}^{m, m+1} \sim \omega_{\mathrm{opt}}^{m+1, m}$, where $A_{m, m+1}$ has a maximum. This maximum corresponds to the situation where the two coupling modes have turning point positions within (or close to) the thermocline layer. In this case, pairs of coupling modes have approximately the same scale of interference beating (or mode cycle distance) $D_{\text {opt }}$ $=2 \pi /\left|\Delta q_{m+1, m}\right|_{\omega=\omega_{\text {opt }}^{m}} \approx$ const.

The authors apply this model to examine our hypothesis with the experimental data.

\section{Intensity temporal fluctuations}

Let us consider the variability of the sound intensity at a fixed frequency (spectral intensity) $I_{\omega}(T)=\left|P_{\omega}(T)\right|^{2} / 2 \rho c$ during an interval $\Delta T$ (i.e., $0<T<\Delta T$, where $\Delta T$ can be on the order of a few hours, when the waveguide parameters and IS field's characteristics are supposed to be constant). The authors thus consider the spectrum of intensity fluctuations, determined by the equation

$$
G(\omega, \Omega)=\int_{0}^{\Delta T} \delta I_{\omega}(T) e^{i \Omega T} d T
$$

where

$$
\delta I_{\omega}(T)=I_{\omega}(T) /\left\langle I_{\omega}\right\rangle-1, \quad\left\langle I_{\omega}\right\rangle=\frac{1}{\Delta T} \int_{0}^{\Delta T} I_{\omega}(T) d T
$$

The function $|G(\omega, \Omega)|$ can be shown in a plot with the vertical axis denoting sound frequency $\omega$ and water column fluctuation frequency $\Omega$ on the horizontal axis. Values of $|G|$ can be shown in a color scale on this plane. The authors denote this picture as a "frequency-frequency $(f F)$-diagram."

Figure 2(b) is from the SWARM95 experimental data ${ }^{1}$ showing the fluctuation spectrum versus the sound frequency spectrum. In this case $\Delta T$ is $1 \mathrm{~h}$ (i.e., 19:00-20:00 GMT on August 4,1995$)$, and the discrete set $T_{i}$ corresponds to geotimes for 60 broadband pulses, which were emitted every minute $(i=1,2, \ldots, 60)$. In this diagram there exists a predominating frequency of fluctuations in the range $\sim 5 \pm 2 \mathrm{cph}$ for the entire sound frequency band [see arrow 
in Fig. 2(b)]. To explain this feature, the authors use the modal decomposition shown in Eqs. (2) and (3) as follows:

$$
G(\omega, \Omega)=\sum_{m, l, n, k} G_{m l k n}(\omega, \Omega)
$$

where each member of the sum is

$$
G_{m l k n}(\Omega, \omega)=\frac{\Delta T}{r \sqrt{q_{m} q_{n}}} S_{m l} S_{n k}^{*} \psi_{l}\left(z_{s}\right) \psi_{k}^{*}\left(z_{s}\right) \psi_{m}(z) \psi_{n}^{*}(z) \frac{\sin \theta}{\theta} \exp \left[i\left(r \Delta q_{m n}+\theta\right)\right] .
$$

Here, $\theta=\theta_{m l k n}=0.5 \Delta T\left[\Omega-\left(\Omega_{m l}-\Omega_{n k}\right)\right]$ and $\Omega_{m l}=v\left|\Delta q_{l m}\right|$.

Equation (9), as a function of frequency $(\omega)$, has maxima at the points $\Omega-\left(\Omega_{m l}\right.$ $\left.-\Omega_{n k}\right)=0$.

In the spectrum of intensity oscillations, the authors observe many spectral lines corresponding to the aforementioned differences. The positions of these spectral lines will depend on sound frequency, due to the frequency dependence of the modal eigenvalues. These differences are determined by the dispersion curves $q_{l}(\omega)$ of the separate modes. Their vertical shape depends on the sound speed profile, the overall depth, and the sound speed in the bottom (i.e., the general waveguide properties). The amplitude (or intensity) of each spectral line also depends on frequency and mode number. In particular, in accord with the fact that the main contribution is provided by adjacent modes (the values $A_{m, m+1}$ are maximal in the sum), the spectral line with maximal intensity occurs at the frequency $\Omega=\Omega_{m, m+1}$ or $\Omega=v\left|\Delta q_{m, m+1}\right|$. In Fig. 2(a), the authors show the result of a calculation of the spectrum of intensity fluctuations $G(\omega, \Omega)$ for different sound frequencies, using a color scale (normalized here and in all figures from 0 to 1 ).

For these calculations, the authors take waveguide parameters corresponding to the experiment in Ref. 1. Specifically, the depth of the source is $\sim 12 \mathrm{~m}$, the water depth at the receiver array is $\sim 85 \mathrm{~m}$, and the velocity of the perturbation along the acoustic track is $v$ $\sim 1.1 \mathrm{~m} / \mathrm{s}$. This value of $v$ provides the best correspondence between the theory in Fig. 2(a) and the experimental data in Fig. 2(b). One can see that the maxima in these spectra are found on the curves $v\left|\Delta q_{m, m+1}\right|=$ const, shown by thin yellow lines in both figures. Every slice of these curves by a horizontal straight line gives the spectrum of the fluctuations for a given sound frequency. The authors see that in these spectra there are many maxima, with the most significant one corresponding approximately to the fluctuation frequency $\Omega_{\mathrm{opt}}=v\left|\Delta q_{m, m+1}\right|_{\omega=\omega_{\mathrm{opt}}^{m}}$ [see arrow in Fig. 2(a)]. However, for different sound frequencies, this predominant frequency of oscillation is provided by different pairs of modes. For example, near $\omega=\omega_{\mathrm{opt}}^{1} \sim 50 \mathrm{~Hz}, \Omega_{\mathrm{opt}}$ $=\Omega_{12}$, whereas near $\omega=\omega_{\mathrm{opt}}^{2} \sim 100 \mathrm{~Hz}, \Omega_{\mathrm{opt}}=\Omega_{23}$, etc.

The value of the dominant fluctuation frequency is $\Omega_{\mathrm{opt}} \sim 13 \times 10^{-4} \mathrm{~Hz}$ (i.e., $\sim 5-6 \mathrm{cph}$ ). Existence of this dominant frequency of intensity fluctuations is the result of the previously mentioned constant length scale for the interference beating of pairs of modes at optimal frequencies. Rough estimates from ray theory for the cycle distance of a ray tangent to the thermocline layer give $D_{\mathrm{opt}} \sim 700-800 \mathrm{~m}$, corresponding to the above frequency, $\sim 5 \mathrm{cph}$. The authors also note that the estimated velocity of the soliton, $v_{s} \sim 0.7 \mathrm{~m} / \mathrm{s}$, is in accord with observations in this area. ${ }^{1,3}$

\section{Arrival time fluctuations}

The group velocity of an individual mode is $v_{l}^{\mathrm{gr}}=\left(d q_{l} / d \omega\right)^{-1}$. If the length of the acoustic track is $L$, then arrival time of a non-coupling mode is $t_{l}=L / v_{l}^{\text {gr }}$. Because group velocities of individual modes depend on frequency, arrival time is also function of frequency, $t_{l}=t_{l}(\omega)$. A set of these functions (or curves in the TF-plane) for different mode numbers is classically referred to as a "TF-diagram" and is often used in pulse propagation analysis. If a propagating mode $l$ meets a soliton at distance $R$, then after their interaction, a newly created set of modes is generated. The "additional" modes with index $m$ propagate after leaving the soliton with their own group velocities. Their arrival time $t_{l m}$ can be estimated as 


$$
t_{l m}=\frac{R}{v_{l}^{\mathrm{gr}}}+\frac{L-R}{v_{m}^{\mathrm{gr}}} .
$$

Thus for the TF-diagram the authors have additional curves $t_{l m}(\omega)$. For uniqueness of notation, the authors denote the arrival time of the uncoupled mode as $t_{l l}$. The frequency and modal dependencies of arrival time can be different, and this is determined by the specific waveguide parameters. For typical conditions (including those of the SWARM95 experiment), for a given frequency $v_{1}^{\mathrm{gr}}>v_{2}^{\mathrm{gr}}>\ldots$, and correspondingly $t_{11}<t_{22}<\ldots$. For the newly created modes, the authors have somewhat more complex relationships between the arrival times and the positions of the additional curves on the TF-diagram. A simpler situation results if the authors take into account the interaction between adjacent modes only. In this case $t_{11}<t_{12}, t_{21}<t_{22}<\ldots$. The relationship between $t_{12}$ and $t_{21}$ (as well as between $t_{l m}$ and $t_{m l}$ ) depends on the position of the soliton (on $R$ ) and on the relationship between the group velocities $v_{1}^{\mathrm{gr}}$ and $v_{2}^{\mathrm{gr}}$.

It is noted that the amplitude of the additional pulse, as per Eq. (3), depends on frequency and distance $R$. It fluctuates as a function of position of the soliton $R$ or as a function of time $T$. This situation can also be described via the TF-diagram, if the authors introduce information about the amplitude of a mode at a given frequency using color gradation. These diagrams are shown in Fig. 3. In Fig. 3(a), the results of theoretical calculations for our model of the SWARM95 waveguide are shown, where the authors assumed a definite position of the soliton. Amplitudes of the modes are denoted using color gradation. The authors can see that, in accordance with our theory, the authors have the most significant amplitude of the additional modes near the optimal sound frequencies, as introduced earlier. Due to the existence of an optimal ray cycle distance $D_{\mathrm{opt}}$, the authors can introduce an optimal group velocity $v_{\mathrm{opt}}^{\mathrm{gr}}$ for each mode, having maximal coupling with its neighbors. Arrival times for these optimal frequencies are also almost the same, $t_{\mathrm{opt}} \sim L / \nu_{\mathrm{opt}}^{\mathrm{gr}}$ (in our case $v_{\mathrm{opt}}^{\mathrm{gr}} \sim 1463 \mathrm{~m} / \mathrm{s}$ ), which is pointed out by the arrow in Figs. 3(a) and 3(b). In Fig. 3(b) the authors show results of the processing of one pulse received in the SWARM95 experiment. The authors also see, in these experimental data, a similar location for the arrival times of modal pulses, which undergo the most significant interaction with each other, and give the corresponding point in the TF-diagram.

\section{Correlation function}

The temporal correlation function for intensity at fixed frequency is the subject of many studies (see, for example, Ref. 4). It can be introduced as

$$
\Gamma_{\omega}(\tau)=\int_{0}^{\Delta T} I_{\omega}(T) I_{\omega}(T-\tau) d T
$$

If the temporal variability is a result of IS motion with a constant velocity, $T=R / v$, then the authors can connect correlation range and correlation time via $\tau=\Delta R_{p} / v$, and therefore the authors can work with the correlation functions $\Gamma_{\omega}\left(\Delta R_{p}\right)$. The range dependent correlation function is often created theoretically due to lack of experimental data that is usually not readily available. However, the temporal correlation function can be constructed more simply from a single point measurement of temporal sequence of received pulses. The temporal correlation length and spatial correlation length are related with each other by the speed of perturbations in the waveguide. One can be obtained from another by using the speed of the IS.

The frequency dependent range correlation function, calculated within the framework of our model, is shown in Fig. 4(a). It shows the correlation between two different positions of the IS. The connection between the range and time correlations can be established if the authors know the velocity of the IS. However, because the authors do not know the velocity experimentally, the authors will construct this function in the model using range. The correlation length denoted in this figure is determined using the half maximum value shown in black. The authors note a comparatively weak decrease in the correlation length with increasing frequency. The value $\Delta R_{p}$ is estimated at about $80-110 \mathrm{~m}$ over a rather broad frequency band. The reason for 
this weak frequency dependence of the correlation length is that only the modes turning tangent to the thermocline give a significant contribution to the range dependent fluctuations. They have close "ray cycles," and a moving soliton creates interaction mostly between these modes. Thus, in range fluctuations, there is a predominant length scale (the ray cycle distance of the above mentioned rays), which does not depend (or depends weakly) on frequency.

If the authors now consider the temporal correlation function, then given a constant velocity of the moving perturbation, the authors have a weak frequency dependence of the correlation time and also a connection between the correlation length and the correlation time. The correlation length depends mainly on the basic waveguide parameters. Thus the correlation time is sensitive only to the waveguide parameters and to the soliton velocity.

The authors can estimate the velocity of the perturbation, which produces the mode coupling. If the authors take the correlation length from theory [Fig. 4(a)] and the correlation time from experiment [Fig. 4(b)], $v=\Delta R_{p} / \tau \sim 1.1-1.2 \mathrm{~m} / \mathrm{s}$. As was mentioned above, the relevant velocity of the soliton is the projection of its intrinsic velocity on the direction of propagation of solitons (thus the authors multiply by $\sin \alpha \sim 0.64$ ), giving $v_{s} \sim 0.7-0.8 \mathrm{~m} / \mathrm{s}$. This result is in good agreement with experimental data ${ }^{1}$ and with the results of Sec. 3 .

\section{Conclusion}

The mechanism governing the intensity temporal fluctuations for large angle $\left(\sim 40^{\circ}\right.$ in SWARM95 data) between the acoustic track and IS crest can be explained by specific features of mode coupling initiated by IS in shallow water. This is in contrary to the case of small angle $\left(\sim 5^{\circ}\right)$, which was shown to be governed by horizontal refraction. ${ }^{3}$

In shallow water the sound speed perturbations (or IS) are usually concentrated in the thermocline region. Therefore, most significant mode coupling takes place between adjacent modes, at some optimal frequencies $\omega_{\mathrm{opt}}^{m}$ (Fig. 2). Here, the acoustic ray turning points are found within (or near) the thermocline region. A pair of modes at the optimal sound frequency gives the most significant contribution to the temporal fluctuations. For different frequency bands, there are other pairs giving the predominant contribution to the fluctuations. However, they have approximately the same interference beat length (or ray cycle) $D_{\mathrm{opt}}$, and provide the predominant frequency of intensity fluctuations $\Omega_{\text {opt }}$ (for the SWARM95 experiment $\Omega_{\text {opt }} \sim 5 \mathrm{cph}$ ).

Strongly interacting modes have close group velocities, approximately $v_{\mathrm{opt}}^{\mathrm{gr}}$ (each pair at different frequencies) and correspondingly close arrival times $t_{\text {opt }}$ (Fig. 3). In particular, good mode separation for narrowband pulses is possible only away from this arrival time.

The optimal parameters including the correlation time (or equivalently the correlation length) are determined mostly by the properties of the unperturbed waveguide.

\section{Acknowledgments}

The authors are grateful for the support provided by RFBR Grant No. 06-05-64853 and ONR Grant Nos. N00014-01-1-0114 and N00014-04-10146 for this research.

\section{References and links}

${ }^{1}$ M. Badiey, Y. J. Mu, J. F. Lynch, R. Apel, and S. N. Wolf, "Temporal and azimuthal dependence of sound propagation in shallow water with internal waves," IEEE J. Ocean. Eng. 27, 117-129 (2002).

${ }^{2}$ M. Badiey, B. Katsnelson, J. Lynch, S. Pereselkov, and W. Siegmann, "Measurement and modeling of 3-D sound intensity variations due to shallow water internal waves," J. Acoust. Soc. Am. 117, 613-625 (2005).

${ }^{3}$ S. D. Frank, M. Badiey, J. Lynch, and W. L. Siegmann, "Analysis and modeling of broadband airgun data influenced by nonlinear internal waves," J. Acoust. Soc. Am. 116, 3404-3422 (2004).

${ }^{4}$ T. F. Duda, "Temporal and cross-range coherence of sound traveling through shallow-water nonlinear internal wave packets," J. Acoust. Soc. Am. 119, 3717-3725 (2006). 